\title{
Study on SLM manufacturing of teeth used for dental tools testing
}

\author{
George Razvan Buican ${ }^{1,}$, Gheorghe Oancea ${ }^{1}$, and Rui F. Martins ${ }^{2}$ \\ ${ }^{1}$ Transilvania University of Brasov, Faculty of Technological Engineering and Industrial \\ Management, Department of Manufacturing Engineering, Mihai Viteazu, No. 5, Brasov, Romania \\ ${ }^{2}$ Universidade Nova de Lisboa, Faculty of Sciences and Technology, UNIDEMI, Campus de \\ Caparica, 2829-516 Caparica, Portugal
}

\begin{abstract}
In this article are studied the possibilities of manufacturing human teeth using the Selective Laser Melting (SLM) process. The human teeth are a biological construction, and because of this, they have a very complex structure with external and internal complex surfaces, unique for each person. To manufacture such structures, even using additive technologies, it is important to find the right manufacturing strategy. In this study are presented three strategies to manufacture the first molar using a SLM process and ANSI $316 \mathrm{~L}$ stainless steel as the building material. The best strategy to manufacture the teeth is chosen, and once build they can be used to test dental tools.
\end{abstract}

\section{Introduction}

It is well-known that nowadays the additive manufacturing process is used in different domains such as: aerospace industry, automotive industry, clothing industry, medicine and even entertainment industry [1-5].

The paper studies the manufacturing possibilities of two human teeth, from stainless steel powder, using the additive manufacturing technologies available on the SLM $250 \mathrm{HL}$ machine.

The 3D models of the two teeth were realized in the CAD software SolidWorks ${ }^{\circledR}$. In order to better design the teeth including the mesiobuccal root canal, with a $0.9 \mathrm{~mm}$ diameter, two X-Ray images, from a maxillary molar morphology study [6], were used, namely the buccolingual and mesiodistal views.

According to the Fig.1., in order to manufacture these teeth the following main steps need to be performed:

- generating the STL file from a CAD model;

- processing the STL file and generating the machine FAB file with the building supports and construction parameters;

- using the machine FAB file to manufacture the 3D object.

In Fig.2. and Fig.3. the 3D representation of the mandibular molar modelled in SolidWorks ${ }^{\circledR}$ is shown.

*Corresponding author: buican.george@,unitbv.ro 


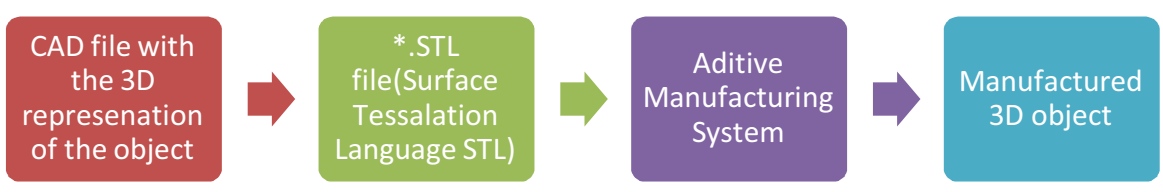

Fig. 1. Steps in creating a 3D object

In the first and second step, the STL file generated by the CAD software is loaded into Magics ${ }^{\circledR}$. Here, the $3 \mathrm{D}$ construct is checked for surface tessellation errors like Manifold leaks, intersecting faces, degenerate faces and empty spaces. Once the file is free of errors the most important step is performed. In this stage, the positioning of the object in relation to the manufacturing table and the building supports is realized [7].

The positioning of the manufacture part and the building supports have such a high importance, because, depending on the choices that are taken, the part will be completely manufactured or will become a failure. Furthermore, the positioning solution influences the mechanical properties and the residual stresses of the finished part, and many other parameters.

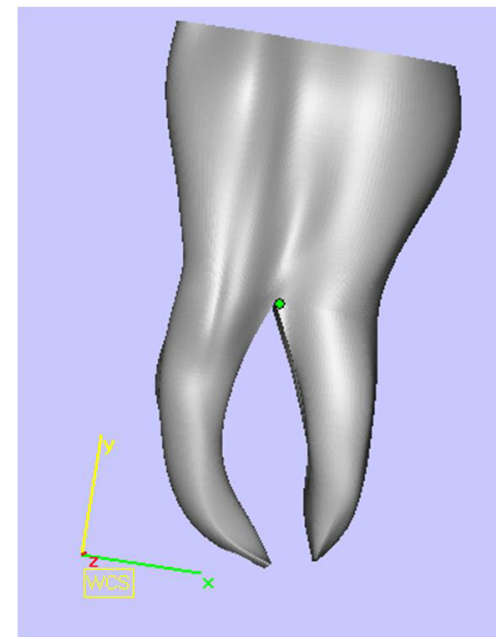

Fig. 2. Mandible molar - 3D view.

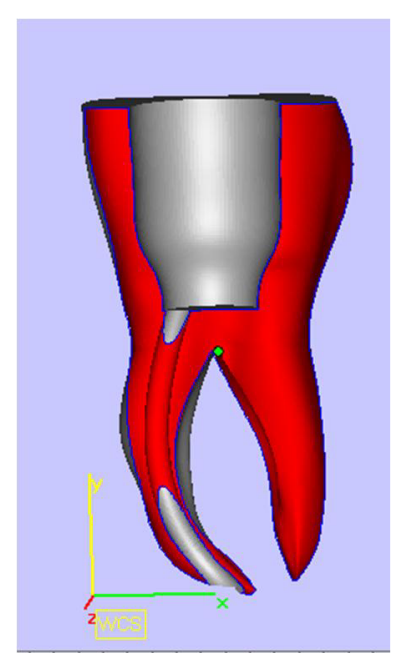

Fig. 3. Mandible molar - section.

Effects which are influenced by the part positioning solution are [8-11]:

- outer surface of the finished part (including the roughness);

- internal stresses;

- mechanical properties;

- material consumption;

- detachment of the part from the building table;

- possibility to build or not internal features;

- number of build parts;

- overall aspect;

- and even the maximum height of the manufactured object.

A lot of parameters are influenced by the positioning solution and, because of this, three solutions for the positioning problem are taken into account (there are many other 
solutions, but we stopped the algorithm of selection when the outcome was the most satisfactory). The considered solutions for the positioning problem are:

- mandible molar is positioned parallel with the building table (Fig.4., Fig.5., Fig.6. and Fig.7.);

- mandible molar root is positioned parallel with the building table (Fig.8. and Fig.9.);

- mandible molar crown is positioned parallel with the building table(Fig.10. and Fig.11.).

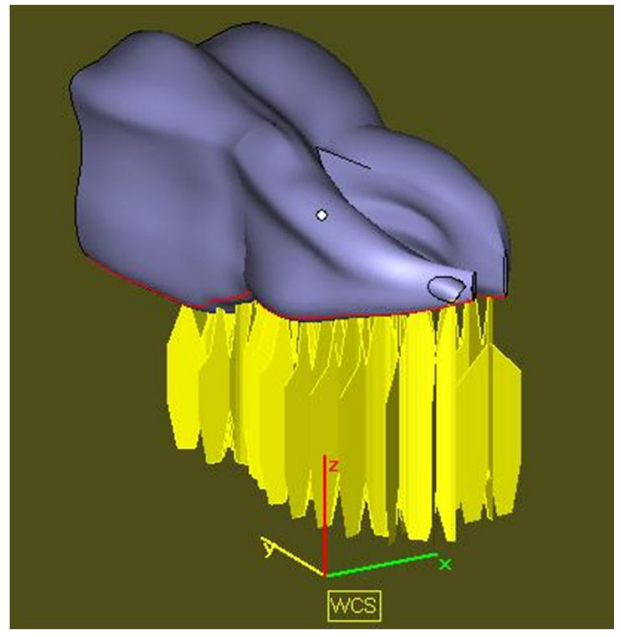

Fig. 4. First case - Mandible molar with construction supports, 3D isometric view.

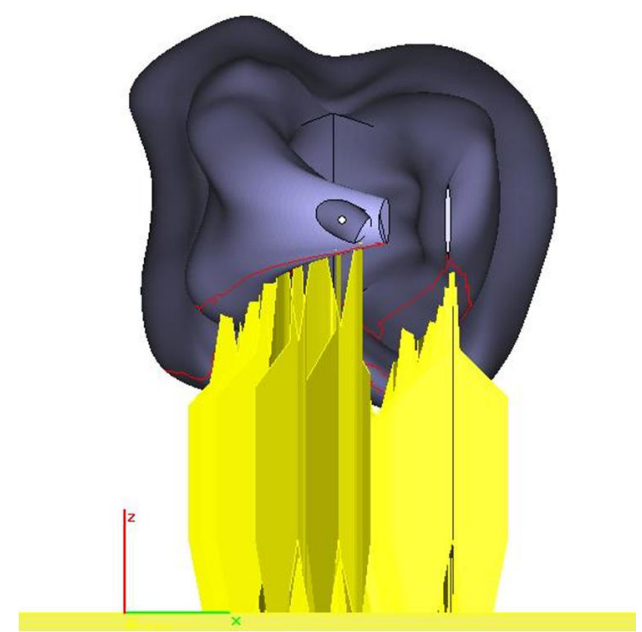

Fig. 5. First case - Mandible molar with construction supports, back view.

\section{Teeth manufacturing - positioning solutions}

\subsection{First case - the mandible molar is positioned parallel with the building table}

As the first case of positioning, for the mandible molar, it is considered the alignment of the teeth in a parallel direction with the building table (Fig.4., Fig.5., Fig.6. and Fig.7.). In this positioning solution, the construction supports must be attached perpendicular to the exterior face of the teeth that is parallel to the building table.

In trying using this positioning solution to manufacture the part were identified a series of disadvantages:

- After manufacturing the tooth, on the surface with the attached building supports it has to be applied a second machining operation to obtain a smooth surface. The surface has irregularities generated by leftover material from the building supports. But, because this surface is a reproduction of an organic growth, the shape is an irregular one and thus very hard to retouch.

- Because the final 3D object is hollow inside (it has cavities) the Magics ${ }^{\circledR}$ software attaches supports even on the interior of the part (Fig.7.). To remove the interior supports is an even more difficult operation, an operation that needs to employ special tools, thus raising the complexity of the manufacturing process and the overall costs.

- Also, the attached supports, from the exterior surface, are not enough to maintain the part fixed until the end of building process, and thus a misconstruction could occur. 


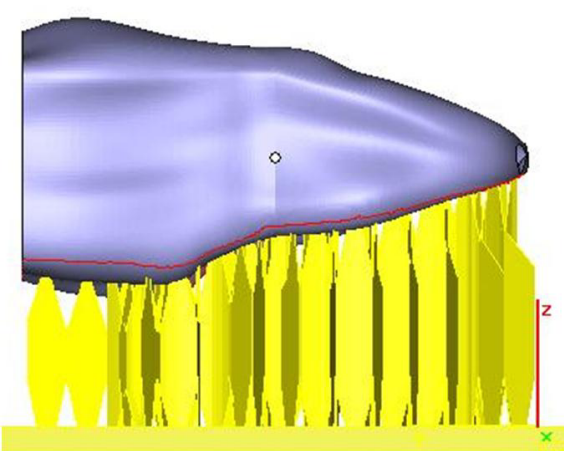

Fig. 6. First case - Mandible molar with construction supports, 3D side view.

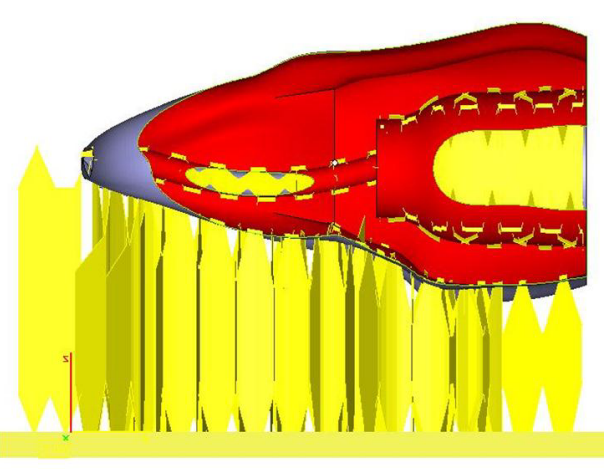

Fig. 7. First case - Mandible molar with construction supports, section.

Taking into account all drawbacks presented above, this positioning solution is considered only a theoretical one, and thus it is not used to create a real object.

\subsection{Second case - the tip of the mandible molar root is positioned parallel with building table}

The second positioning solution has the construction supports attached on the tip of the teeth root (Fig.8. and Fig.9.). To a first glance this could be considered as the best positioning solution because of the belief that there is no need for post processing operations.

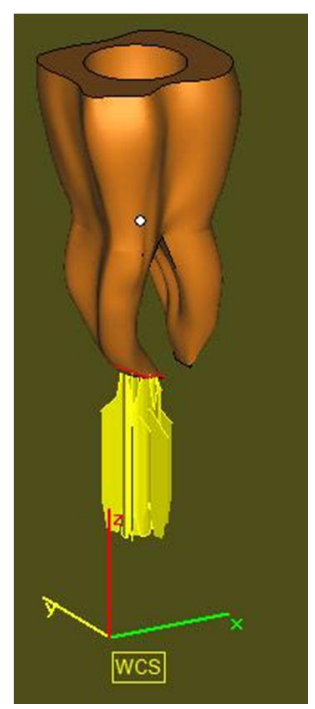

Fig. 8. Second case - Mandible molar with construction supports, 3D Isometric view.

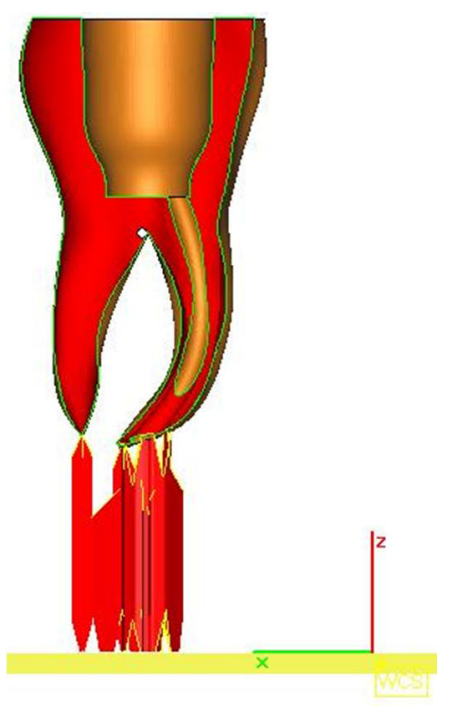

Fig. 9. Second case - Mandible molar with construction supports, section.

The area of contact between the manufacturing supports and the finished part is the smallest of all the cases, but it is well known that the process of selective laser melting is a manufacturing process that inserts in the finished part a lot of residual stresses and tensions 
due to the subsequent melting and cooling cycles [4-5,8-11]. These melting and cooling cycles are due to the way the technology works.

The selective laser melting process is an additive manufacturing method, and a layer based technology. Thus it consists in building the part in layers of fixed thickness, which are stacked one upon another, and then melted by using an energy source.

In the considered case, the metal powder (ANSI 316-L stainless steel) is laid in a thick layer of $30 \mu \mathrm{m}$ on the building plate, and then the laser melts a predefine contour, that represents a 2D section from the part. On top of this layer, the machine lays another layer of material and the process is repeated until the part is finished.

Keeping in mind the way this technology works, and the stresses and tensions that are inserted in the part because of the heating and cooling cycles, it is concluded that the building supports in this positioning solution are not sufficient to take this job to the end. This case is a theoretical one.

\subsection{Third case - the mandible molar crown is positioned parallel to the building table}

The third positioning solution lay the teeth with the crown parallel to the building plate. In this position the supports are attached to the teeth crown, that, in the considered case, is a flat surface, and thus provide a sufficient surface for the part to be fixed during the entire manufacturing process (Fig.10. and Fig.11.).

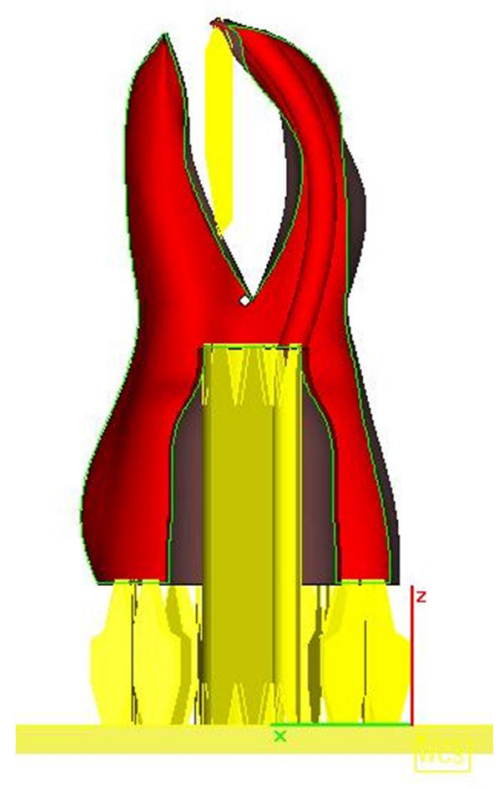

Fig. 10. Third case - Mandible molar with all construction supports, section.

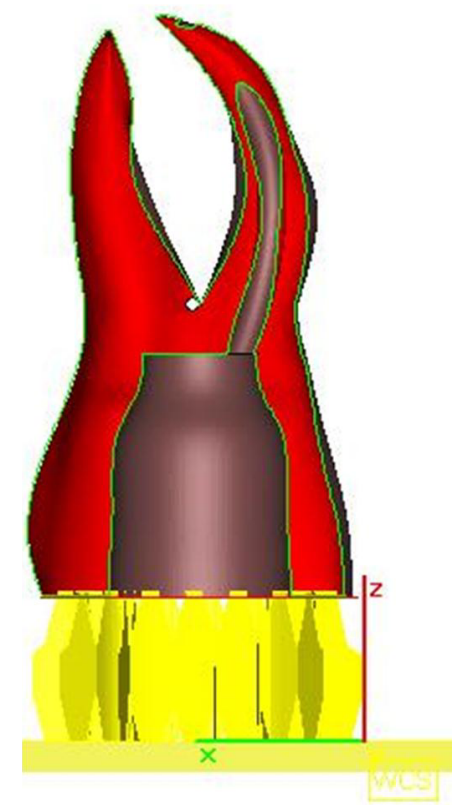

Fig. 11. Third case - Mandible molar with crown construction supports, section.

Also, on this position solution, building supports can be attached to the interior of the teeth, to further sustain the part. This is possible because here there is a flat surface.

Additionally, there is a need to attach a point support to assist the root of the teeth during the manufacturing process, and this is needed because the root has a very big curvature. 


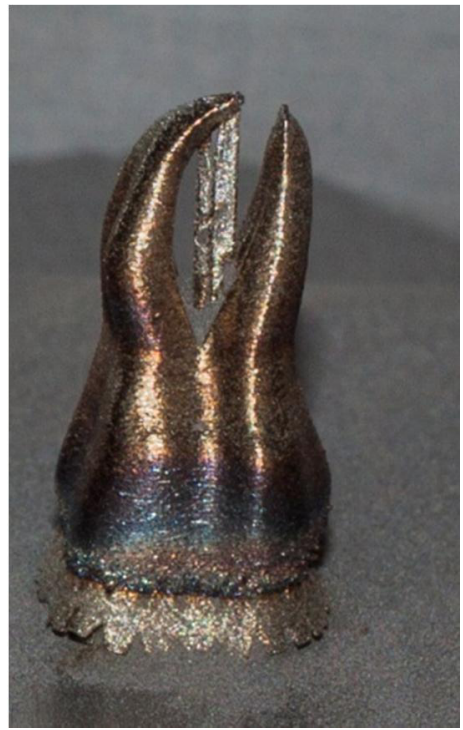

Fig. 12. Mandible molar attached to building plate, front view.

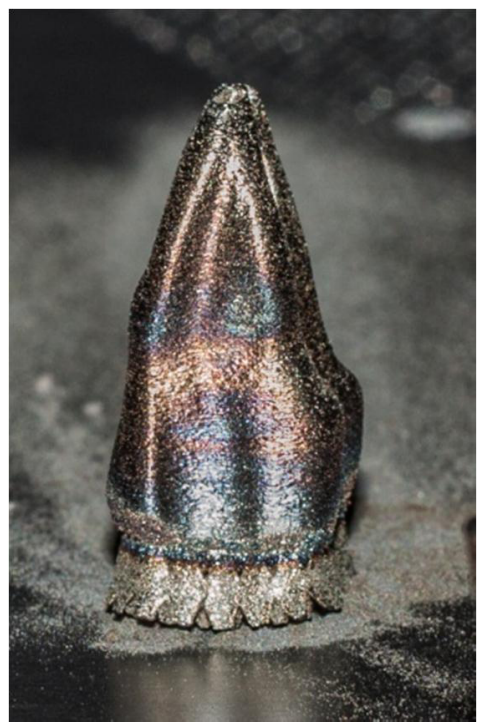

Fig. 13. Mandible molar attached to building plate, side view.

Because the building supports are attached to a plan surface, the post processing is much easier to be made. In the same time the part detaching from the building table is much improved.

The contact area between the building supports and teeth crown is situated in the same plane, but has a circular cross-section, thus providing more strength and resilience against the stresses that appear during the manufacturing process.

This case is considered the best solution for the positioning and it will be used in practice, to manufacture the teeth.

\section{Teeth Manufacturing on SLM $250 \mathrm{HL}$}

The mandible molar was manufactured on the SLM 250-HL equipment using a layers size of $30 \mu \mathrm{m}$. In order to manufacture the teeth, the positioning solutions, together with the building supports are loaded in AutoFab ${ }^{\circledR}$ and the final file with the manufacturing parameters is created.

Teeth are manufactured using a stainless steel ANSI 316-L powder. This material is laid as a thin layer of $30 \mu \mathrm{m}$ on top the building plate, then the layers generated with AutoFab®, in the *.fab file, are melted in the metal layer using different laser powers (Fig.12, Fig.13, Fig.14., Fig.15., Fig.16. and Fig.17.).

In order to build this part it was used a strategy with different working parameters. This strategy was established according to the areas of the parts: contour, volume, overhang, skin and building supports. The meanings of the areas and the laser powers used in the manufacturing process on each area are:

- contour - it is the contour of the 2D section; the laser power is $100 \mathrm{~W}$;

- volume is interior of the $2 \mathrm{D}$ section and it is more than just a contour is an internal surface. Here the laser power is $175 \mathrm{~W}$, but in addition to the rest of the building regimes here it was defined even a path for the laser( in this case chess board, rotated to 45 degrees on each layer); 
- overhang is the area where the building supports and the actual part meet. Here the used laser power was $175 \mathrm{~W}$;

- skin is the outer layer of the finished part, and thus has a different set of parameters, parameters that give a smoothness surface. In this area the laser power is also $175 \mathrm{~W}$, but the laser speed is changed.

- building supports are manufactured, using a laser power of $100 \mathrm{~W}$, and a different pattern for the path of the laser. In this area the laser spot is at its smallest value, because the building supports can be compared to a thin wall part.

Once the parts are finished, together with the building plate they are placed in an oven for tensions eliminating process. After that they are removed from the plate and post processed to remove the building supports.

A second post processing operation can be done using a sand blasting machine to generate a fine exterior surface. This last method is also good for removing scorching marks generated by an overheating during the manufacturing process.

The mandible molar after the post processing operation is presented in the figures 16 and 17.

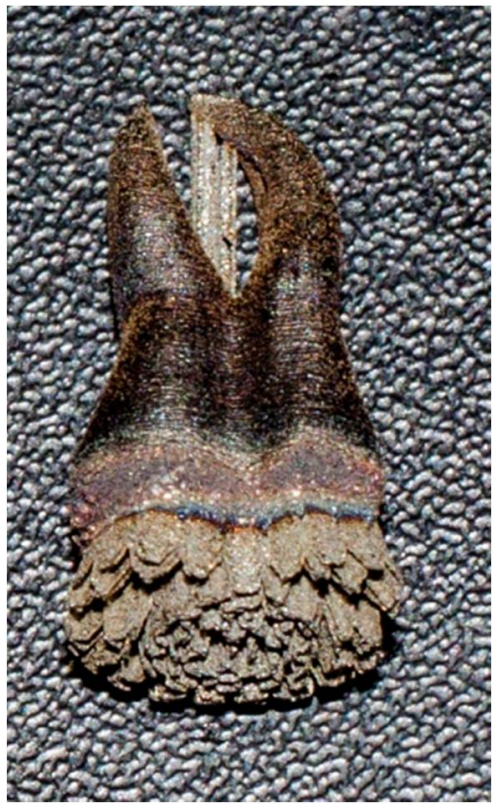

Fig. 14. Mandible molar detached from building plate, front view.

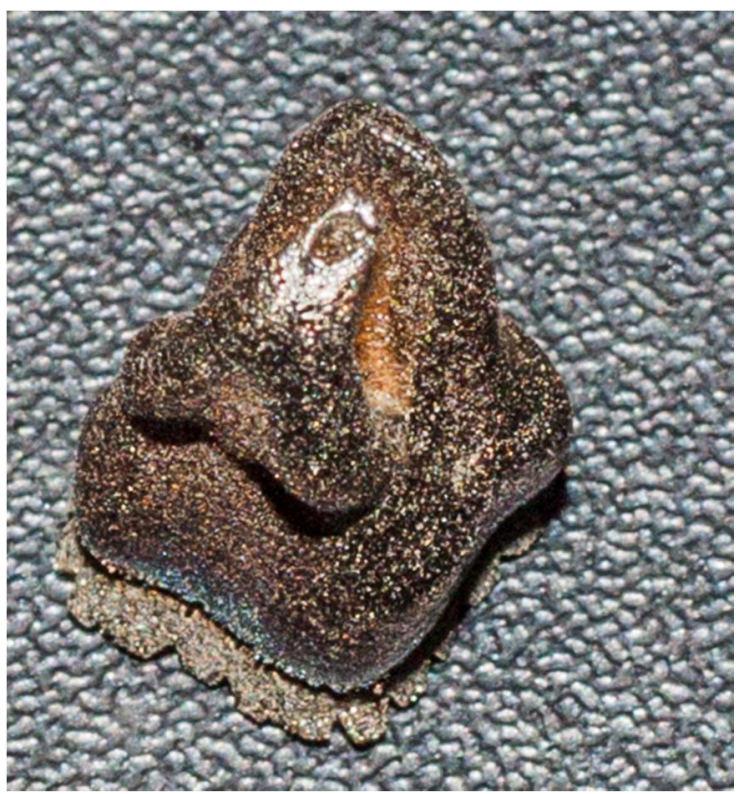

Fig. 15. Mandible molar - detached from the building table, top view.

From these studies, it is revealed that the possibility to manufacture the teeth, all the way to the last layer, is inverse proportional with the complexity of the building supports and positioning solution. This means that the intersection between the surface of the object and the surface generated by the tips of the building supports needs to be as close as possible to a plane. 


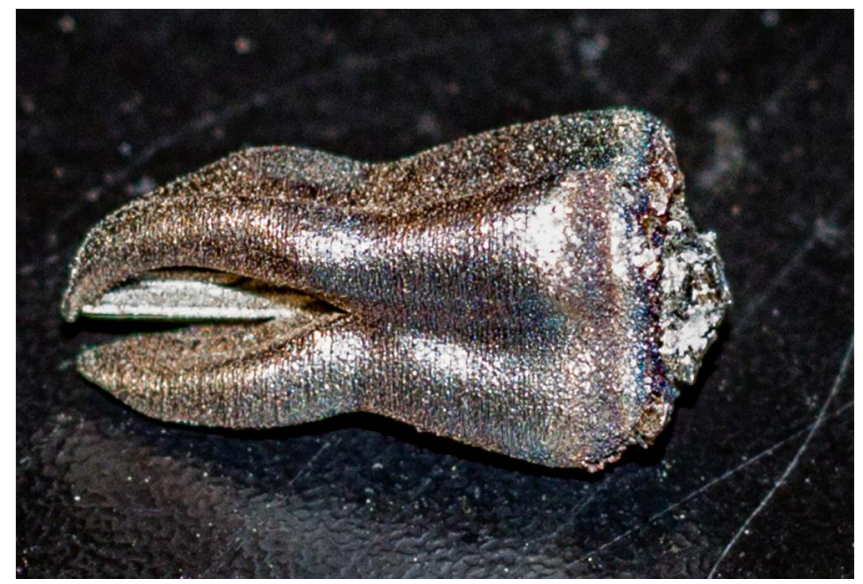

Fig. 16. Mandible molar - detached from the building table with the supports removed, front view.

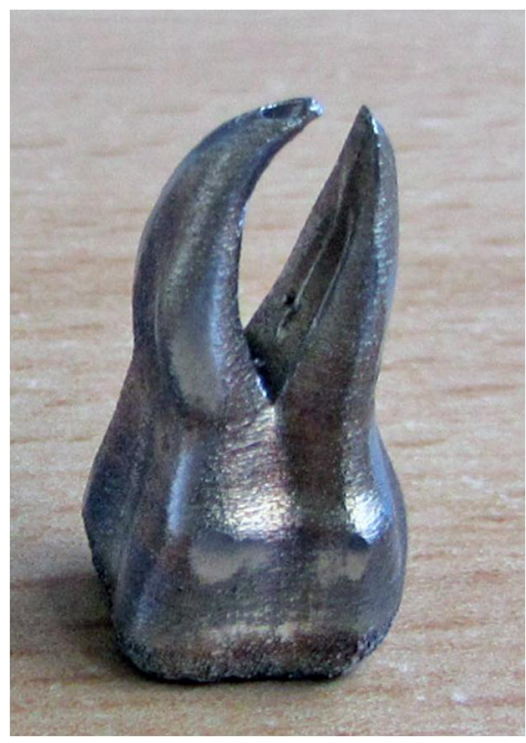

Fig. 17. Mandible molar - finished part, front view.

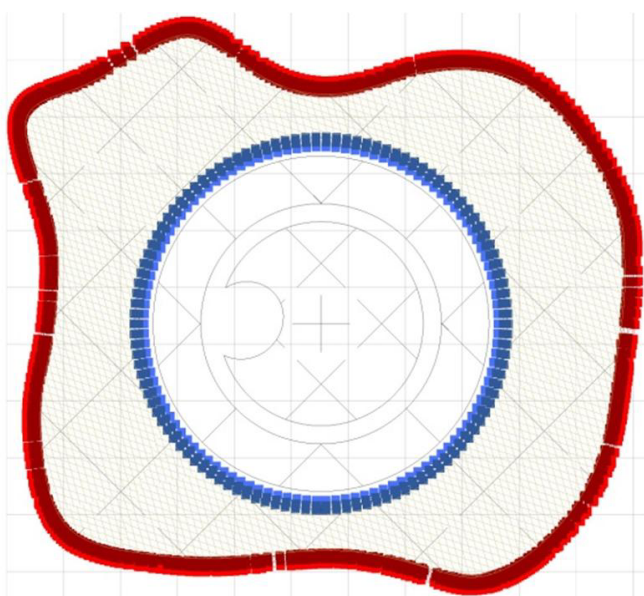

Fig. 18. Generated 2D shape from the intersections.

The intersection between the planes of the building supports tips and the surface on which they are attached, needs to generate a 2D shape that is, either parallel to the building table (for small enough objects), or inclined with a certain degree, (for bigger objects).

By studying three positioning solutions, it was identified three cases of generated shapes:

- in the first case the generated surface, from the intersection, was a 3D shape;

- the second case had generated a planar surface, but it was not a shape but rather a collection of points;

- in the third, and last case, the generated shape was a 2D shape (Fig.18.). 


\section{Conclusions}

By studying the possibility to manufacture human teeth it was identified the possibility to reproduce organic shapes, with high structural complexity, by the means of additive manufacturing, in this case the selective laser melting technology was used. As it can be seen the organic shapes are very complex, each one unique. They have a rough overall shape, but each individual piece has its one distinctive features.

In order to build complex organic shapes with additive manufacturing it is imperative to be able to convert these shapes in 3D data, and only then the technology is able to manufacture these objects. But this is not enough, in order to secure the manufacturing of the teeth, it is necessary to further study the positioning of the objects in rapport with the building table and the building supports.

Based on the studies developed by the authors and presented in this paper, it can be concluded that complex organic shapes can be manufacture with metal additive manufacture technology. For some shapes, this being the only possibility, but in order to be able to finish the part there is a need for rigorous post processing. These post processing operations and simulation help in reducing time and money by increasing the likelihood of completely finishing the parts, or finishing the part without major defects. Using a proper strategy for a selective laser melting technology two human teeth were manufactured on SLM HL 250 machine. The manufactured teeth have been used in the testing process of dental tools.

This paper is supported by the Sectoral Operational Programme Human Resources Development (SOP HRD), ID134378 financed from the European Social Fund and by the Romanian Government.

\section{References}

1. D. Leordean, C. Dudescu, T. Marcu, P. Berce, N. Balc, Customized implants with specific properties, made by selective laser melting, Rapid Prototyping Journal, 21, 98104, (2016)

2. S.C. Cosma, N. Balc, D. Leordean, M. Moldovan, M. Dudescu, C. Borzan, Customized Medical Applications of Selective Laser Melting Manufacturing, Academic Journal of Manufacturing Engineering, 13, 24-32, (2015)

3. S.A. Haba, G. Oancea, Digital manufacturing of air-cooled single-cylinder engine block, The International Journal of Advanced Manufacturing Technology, 80 (5), 747 759, (2015).

4. G.R. Buican, G. Oancea, A. Manolescu, Remanufacturing of Damaged Parts Using Selective Laser Melting Technology, Applied Mechanics and Materials, 693, 285-290, (2014)

5. R. Pacurar, N. Balc, F. Prem, Research on how to improve the accuracy of the SLM metallic parts, The 14th International ESAFORM Conference on Material Forming: ESAFORM, 1353 (1), 1385-1390, (2011).

6. C.E. Jerome, R.J. Hanlon, Identifying Multiplanar Root Canal Curvatures Using Stainless-Steel Instruments, Journal of Endodontics, 29, 356-358, (2003)

7. H. W. Wai, Introduction to STL format, (1999);

8. T. Itziar, G. Fermín, Z. Fidel, Z. Fidel, E. Aritz, Study of mechanical properties of AISI 316 stainless steel processed by "selective laser melting", following different manufacturing strategies, The International Journal of Advanced Manufacturing Technology, 51, 639-647, (2010). 
9. G.R. Buican, G. Oancea, C. Lancea, M.A. Considerations Regarding Micro Hardness of Parts Manufactured from 316-L Steel Using SLM Technology, Applied Mechanics and Materials, Some 760, 515-520, (2015)

10. G.R. Buican, G. Oancea, C. Lancea, M.A. Pop, Influence of Layer Thickness on Internal Structure of Parts Manufactured from 316-L Steel Using SLM Technology, Applied Mechanics and Materials, 809-810, 369-374, (2015)

11. S.C. Muresan, PhD Thesis, Researches regarding the mechanical strength of complex metal parts manufactured by selective laser melting, Technical University of ClujNapoca, (2012) 